\title{
Anisotropy and chemical composition of ultra-high energy cosmic rays using arrival directions measured by the Pierre Auger Observatory
}

The Pierre Auger Collaboration

P. Abreu ${ }^{74}$, M. Aglietta ${ }^{57}$, E.J. Ahn ${ }^{93}$, I.F.M. Albuquerque ${ }^{19}$, D. Allard ${ }^{33}$, I. Allekotte ${ }^{1}$, J. Allen ${ }^{96}$, P. Allison ${ }^{98}$, J. Alvarez Castillo ${ }^{67}$, J. Alvarez-Muñiz ${ }^{84}$, M. Ambrosio ${ }^{50}$, A. Aminaei ${ }^{68}$, L. Anchordoqui ${ }^{109}$, S. Andringa ${ }^{74}$, T. Antičić ${ }^{27}$, A. Anzalone ${ }^{56}$, C. Aramo ${ }^{50}$, E. Arganda ${ }^{81}$, F. Arqueros ${ }^{81}$, H. Asorey ${ }^{1}$, P. Assis ${ }^{74}$, J. Aublin ${ }^{35}$, M. Ave ${ }^{41}$, M. Avenier ${ }^{36}$, G. Avila ${ }^{12}$, T. Bäcker ${ }^{45}$, M. Balzer ${ }^{40}$, K.B. Barber ${ }^{13}$, A.F. Barbosa ${ }^{16}$, R. Bardenet ${ }^{34}$, S.L.C. Barroso ${ }^{22}$, B. Baughman ${ }^{98}$, J. Bäuml ${ }^{39,41}$, J.J. Beatty ${ }^{98}$, B.R. Becker ${ }^{106}$, K.H. Becker ${ }^{38}$, A. Bellétoile ${ }^{37}$, J.A. Bellido ${ }^{13}$, S. BenZvi ${ }^{108}$, C. Berat ${ }^{36}$, X. Bertou ${ }^{1}$, P.L. Biermann ${ }^{42}$, P. Billoir ${ }^{35}$, F. Blanco ${ }^{81}$, M. Blanco ${ }^{82}$, C. Bleve ${ }^{38}$, H. Blümer ${ }^{41,39}$, M. Boháčováá ${ }^{29,101}$, D. Boncioli ${ }^{51}$, C. Bonifazi ${ }^{25,}{ }^{55}$, R. Bonino ${ }^{57}$, N. Borodai ${ }^{72}$, J. Brack ${ }^{91}$, P. Brogueira $^{74}$, W.C. Brown ${ }^{92}$, R. Bruijn ${ }^{87}$, P. Buchholz ${ }^{45}$, A. Bueno ${ }^{83}$, R.E. Burton ${ }^{89}$, K.S. Caballero-Mora ${ }^{41}$, L. Caramete ${ }^{42}$, R. Caruso $^{52}$, A. Castellina ${ }^{57}$, O. Catalano ${ }^{56}$, G. Cataldi ${ }^{49}$, L. Cazon ${ }^{74}$, R. Cester ${ }^{53}$, J. Chauvin $^{36}$, S.H. Cheng ${ }^{99}$, A. Chiavassa ${ }^{57}$, J.A. Chinellato ${ }^{20}$, A. Chou ${ }^{93,96}$, J. Chudoba ${ }^{29}$, R.W. Clay ${ }^{13}$, M.R. Coluccia ${ }^{49}$, R. Conceição ${ }^{74}$, F. Contreras ${ }^{11}$, H. Cook ${ }^{87}$, M.J. Cooper ${ }^{13}$, J. Coppens ${ }^{68,70}$, A. Cordier $^{34}$, U. Cotti6 $^{66}$, S. Coutu ${ }^{99}$, C.E. Covault ${ }^{89}$, A. Creusot ${ }^{33,79}$, A. Criss ${ }^{99}$, J. Cronin ${ }^{101}$, A. Curutiu ${ }^{42}$, S. Dagoret-Campagne ${ }^{34}$, R. Dallier ${ }^{37}$, S. Dasso ${ }^{8,4}$, K. Daumiller ${ }^{39}$, B.R. Dawson ${ }^{13}$, R.M. de Almeida ${ }^{26,20}$, M. De Domenico ${ }^{52}$, C. De Donato ${ }^{67}{ }^{48}$, S.J. de Jong ${ }^{68}$, G. De La Vega ${ }^{10}$, W.J.M. de Mello Junior ${ }^{20}$, J.R.T. de Mello Neto ${ }^{25}$, I. De Mitrii ${ }^{49}$, V. de Souza ${ }^{18}$, K.D. de Vries ${ }^{69}$, G. Decerprit ${ }^{33}$, L. del Peral ${ }^{82}$, O. Deligny ${ }^{32}$, H. Dembinski ${ }^{41,39}$, N. Dhital ${ }^{95}$, C. Di Giulio ${ }^{47,51}$, J.C. Diaz ${ }^{95}$, M.L. Díaz Castro ${ }^{17}$, P.N. Diep ${ }^{110}$, C. Dobrigkeit ${ }^{20}$, W. Docters ${ }^{69}$, J.C. D'Olivo ${ }^{67}$, P.N. Dong ${ }^{110,32}$, A. Dorofeev ${ }^{91}$, J.C. dos Anjos ${ }^{16}$, M.T. Dova ${ }^{7}$, D. D'Urso ${ }^{50}$, I. Dutan ${ }^{42}$, J. $\operatorname{Ebr}^{29}$, R. Enge ${ }^{39}$, M. Erdmann ${ }^{43}$, C.O. Escobar ${ }^{20}$, A. Etchegoyen ${ }^{2}$, P. Facal San Luis ${ }^{101}$, I. Fajardo Tapia $^{67}$, H. Falcke ${ }^{68,71}$, G. Farrar ${ }^{96}$, A.C. Fauth $^{20}$, N. Fazzini ${ }^{93}$, A.P. Ferguson ${ }^{89}$, A. Ferrero ${ }^{2}$, B. Fick ${ }^{95}$, A. Filevich ${ }^{2}$, A. Filipčič ${ }^{78,79}$, S. Fliescher ${ }^{43}$, C.E. Fracchiolla ${ }^{91}$, E.D. Fraenkel ${ }^{69}$, U. Fröhlich ${ }^{45}$, B. Fuchs ${ }^{16}$, R. Gaior ${ }^{35}$, R.F. Gamarra ${ }^{2}$, S. Gambetta ${ }^{46}$, B. García ${ }^{10}$, D. García Gámez ${ }^{83}$, D. GarciaPinto $^{81}$, A. Gascon ${ }^{83}$, H. Gemmeke ${ }^{40}$, K. Gesterling ${ }^{106}$, P.L. Ghia ${ }^{35,57}$, U. Giaccari ${ }^{49}$, M. Giller ${ }^{73}$, H. Glass ${ }^{93}$, M.S. Gold ${ }^{106}$, G. Golup ${ }^{1}$, F. Gomez Albarracin ${ }^{7}$, M. Gómez Berisso ${ }^{1}$, P. Gonçalves ${ }^{74}$, D. Gonzalez ${ }^{41}$, J.G. Gonzalez ${ }^{41}$, B. Gookin ${ }^{91}$, D. Góra ${ }^{41}{ }^{72}$, A. Gorgi ${ }^{57}$, P. Gouffon ${ }^{19}$, S.R. Gozzini ${ }^{87}$, E. Grashorn ${ }^{98}$, S. Grebe ${ }^{68}$, N. Griffith ${ }^{98}$, M. Grigat ${ }^{43}$, A.F. Grillo ${ }^{58}$, Y. Guardincerri ${ }^{4}$, F. Guarino ${ }^{50}$, G.P. Guedes ${ }^{21}$, A. Guzman ${ }^{67}$, J.D. Hague ${ }^{106}$, P. Hansen ${ }^{7}$, D. Harari ${ }^{1}$, S. Harmsma ${ }^{69}$, 70, J.L. Harton ${ }^{91}$, A. Haungs ${ }^{39}$, T. Hebbeker ${ }^{43}$, D. Heck ${ }^{39}$, A.E. Herve ${ }^{13}$, C. Hojvat ${ }^{93}$, N. Hollon ${ }^{101}$, V.C. Holmes ${ }^{13}$, P. Homola ${ }^{72}$, J.R. Hörandel ${ }^{68}$, A. Horneffer ${ }^{68}$, M. Hrabovskýs ${ }^{30,29}$, T. Huege ${ }^{39}$, A. Insolia ${ }^{52}$, F. Ionita ${ }^{101}$, A. Italiano ${ }^{52}$, C. Jarne ${ }^{7}$, S. Jiraskova ${ }^{68}$, K. Kadija ${ }^{27}$, K.H. Kampert ${ }^{38}$, P. Karhan ${ }^{28}$, P. Kasper ${ }^{93}$, B. Kégl ${ }^{34}$, B. Keilhauer ${ }^{39}$, A. Keivani ${ }^{94}$, J.L. Kelley ${ }^{68}$, E. Kemp ${ }^{20}$, R.M. Kieckhafer ${ }^{95}$, H.O. Klages ${ }^{39}$, M. Kleifges ${ }^{40}$, J. Kleinfeller ${ }^{39}$, J. Knapp ${ }^{87}$, D.-H. Koang ${ }^{36}$, K. Kotera ${ }^{101}$, N. Krohm ${ }^{38}$, O. Krömer ${ }^{40}$, D. Kruppke-Hansen ${ }^{38}$, F. Kuehn ${ }^{93}$, D. Kuempel ${ }^{38}$, J.K. Kulbartz ${ }^{44}$, N. Kunka ${ }^{40}$, G. La Rosa ${ }^{56}$, C. Lachaud ${ }^{33}$, P. Lautridou ${ }^{37}$, M.S.A.B. Leão ${ }^{24}$, D. Lebrun ${ }^{36}$, P. Lebrun ${ }^{93}$, M.A. Leigui de Oliveira ${ }^{24}$, A. Lemiere ${ }^{32}$, A. Letessier-Selvon ${ }^{35}$, I. Lhenry-Yvon ${ }^{32}$, K. Link ${ }^{41}$, R. López ${ }^{63}$, 
A. Lopez Agüera ${ }^{84}$, K. Louedec ${ }^{34}$, J. Lozano Bahilo ${ }^{83}$, A. Lucero ${ }^{2,57}$, M. Ludwig ${ }^{41}$, H. Lyberis ${ }^{32}$, M.C. Maccarone ${ }^{56}$, C. Macolino ${ }^{35}$, S. Maldera ${ }^{57}$, D. Mandat ${ }^{29}$, P. Mantsch ${ }^{93}$, A.G. Mariazzi ${ }^{7}$, J. Marin ${ }^{11,57}$, V. Marin ${ }^{37}$, I.C. Maris ${ }^{35}$, H.R. Marquez Falcon ${ }^{66}$, G. Marsella ${ }^{54}$, D. Martello ${ }^{49}$, L. Martin ${ }^{37}$, H. Martinez ${ }^{64}$, O. Martínez Bravo ${ }^{63}$, H.J. Mathes ${ }^{39}$, J. Matthews ${ }^{94,}{ }^{100}$, J.A.J. Matthews ${ }^{106}$, G. Matthiae ${ }^{51}$, D. Maurizio ${ }^{53}$, P.O. Mazur ${ }^{93}$, G. Medina-Tanco ${ }^{67}$, M. Melissas ${ }^{41}$, D. Melo ${ }^{2,53}$, E. Menichetti ${ }^{53}$, A. Menshikov ${ }^{40}$, P. Mertsch ${ }^{85}$, C. Meurer ${ }^{43}$, S. Mićanović ${ }^{27}$, M.I. Micheletti ${ }^{9}$, W. Miller ${ }^{106}$, L. Miramonti ${ }^{48}$, S. Mollerach ${ }^{1}$, M. Monasor ${ }^{101}$, D. Monnier Ragaigne ${ }^{34}$, F. Montanet $^{36}$, B. Morales ${ }^{67}$, C. Morello ${ }^{57}$, E. Moreno ${ }^{63}$, J.C. Moreno ${ }^{7}$, C. Morris ${ }^{98}$, M. Mostafá ${ }^{91}$, C.A. Moura ${ }^{24,50}$, S. Mueller ${ }^{39}$, M.A. Muller ${ }^{20}$, G. Müller ${ }^{43}$, M. Münchmeyer ${ }^{35}$, R. Mussa ${ }^{53}$, G. Navarra ${ }^{57} \dagger$, J.L. Navarro ${ }^{83}$, S. Navas ${ }^{83}$, P. Necesal ${ }^{29}$, L. Nellen ${ }^{67}$, A. Nelles ${ }^{68}$, P.T. Nhung ${ }^{110}$, L. Niemietz ${ }^{38}$, N. Nierstenhoefer ${ }^{38}$, D. Nitz ${ }^{95}$, D. Nosek ${ }^{28}$, L. Nožka ${ }^{29}$, M. Nyklicek ${ }^{29}$, J. Oehlschläger ${ }^{39}$, A. Olinto ${ }^{101}$, P. Oliva ${ }^{38}$, V.M. Olmos-Gilbaja ${ }^{84}$, M. Ortiz ${ }^{81}$, N. Pacheco ${ }^{82}$, D. Pakk Selmi-Dei ${ }^{20}$, M. Palatka ${ }^{29}$, J. Pallotta ${ }^{3}$, N. Palmieri ${ }^{41}$, G. Parente $^{84}$, E. Parizot ${ }^{33}$, A. Parra ${ }^{84}$, R.D. Parsons ${ }^{87}$, S. Pastor ${ }^{80}$, T. Paul ${ }^{97}$, M. Pech ${ }^{29}$, J. Pękala ${ }^{72}$, R. Pelayo ${ }^{84}$, I.M. Pepe ${ }^{23}$, L. Perrone ${ }^{54}$, R. Pesce ${ }^{46}$, E. Petermann ${ }^{105}$, S. Petrera ${ }^{47}$, P. Petrinca ${ }^{51}$, A. Petrolini ${ }^{46}$, Y. Petrov ${ }^{91}$, J. Petrovic ${ }^{70}$, C. Pfendner ${ }^{108}$, N. Phan ${ }^{106}$, R. Piegaia ${ }^{4}$, T. Pierog ${ }^{39}$, P. Pieroni $^{4}$, M. Pimenta ${ }^{74}$, V. Pirronello ${ }^{52}$, M. Platino ${ }^{2}$, V.H. Ponce ${ }^{1}$, M. Pontz ${ }^{45}$, P. Privitera ${ }^{101}$, M. Prouza ${ }^{29}$, E.J. Quel ${ }^{3}$, S. Querchfeld ${ }^{38}$, J. Rautenberg ${ }^{38}$, O. Ravel ${ }^{37}$, D. Ravignani ${ }^{2}$, B. Revenu ${ }^{37}$, J. Ridky ${ }^{29}$, S. Riggi ${ }^{84}{ }^{52}$, M. Risse ${ }^{45}$, P. Ristori ${ }^{3}$, H. Rivera ${ }^{48}$, V. Rizi ${ }^{47}$, J. Roberts ${ }^{96}$, C. Robledo ${ }^{63}$, W. Rodrigues de Carvalho ${ }^{84}, 19$, G. Rodriguez ${ }^{84}$, J. Rodriguez Martino ${ }^{11,52}$, J. Rodriguez Rojo ${ }^{11}$, I. Rodriguez-Cabo ${ }^{84}$, M.D. Rodríguez-Frías ${ }^{82}$, G. Ros ${ }^{82}$, J. Rosado ${ }^{81}$, T. Rossler ${ }^{30}$, M. Roth ${ }^{39}$, B. Rouillé-d'Orfeuil ${ }^{101}$, E. Roulet ${ }^{1}$, A.C. Rovero ${ }^{8}$, C. Rühle ${ }^{40}$, F. Salamida ${ }^{47}, 39$, H. Salazar ${ }^{63}$, G. Salina ${ }^{51}$, F. Sánchez ${ }^{2}$, M. Santander ${ }^{11}$, C.E. Santo ${ }^{74}$, E. Santos ${ }^{74}$, E.M. $\operatorname{Santos}^{25}$, F. Sarazin ${ }^{90}$, B. Sarkar ${ }^{38}$, S. Sarkarr ${ }^{85}$, R. Sato ${ }^{11}$, N. Scharf ${ }^{43}$, V. Scherini ${ }^{48}$, H. Schieler ${ }^{39}$, P. Schiffer ${ }^{43}$, A. Schmidt ${ }^{40}$, F. Schmidt ${ }^{101}$, T. Schmidt ${ }^{41}$, O. Scholten ${ }^{69}$, H. Schoorlemmer ${ }^{68}$, J. Schovancova ${ }^{29}$, P. Schovánek ${ }^{29}$, F. Schröder ${ }^{39}$, S. Schulte ${ }^{43}$, D. Schuster ${ }^{90}$, S.J. Sciutto ${ }^{7}$, M. Scuderi ${ }^{52}$, A. Segreto ${ }^{56}$, M. Settimo ${ }^{45}$, A. Shadkam ${ }^{94}$, R.C. Shellard ${ }^{16,17}$, I. Sidelnik ${ }^{2}$, G. Sigl ${ }^{44}$, H.H. Silva Lopez $^{67}$, A. Śmiałkowski ${ }^{73}$, R. Śmída ${ }^{39,29}$, G.R. Snow ${ }^{105}$, P. Sommers ${ }^{99}$, J. Sorokin ${ }^{13}$, H. Spinka ${ }^{88,93}$, R. Squartini ${ }^{11}$, J. Stapleton ${ }^{98}$, J. Stasielak ${ }^{72}$, M. Stephan ${ }^{43}$, E. Strazzeri ${ }^{56}$, A. Stutz ${ }^{36}$, F. Suarez ${ }^{2}$, T. Suomijärvii ${ }^{32}$, A.D. Supanitsky ${ }^{8,67}$, T. Šuša ${ }^{27}$, M.S. Sutherland ${ }^{94,}{ }^{98}$, J. Swain ${ }^{97}$, Z. Szadkowski ${ }^{73}, 38$,

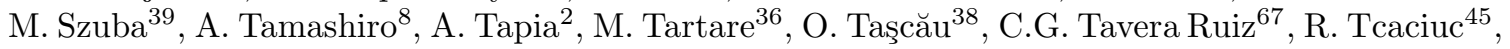
D. Tegolo ${ }^{52,61}$, N.T. Thao ${ }^{110}$, D. Thomas ${ }^{91}$, J. Tiffenberg ${ }^{4}$, C. Timmermans ${ }^{70,68}$, D.K. Tiwari ${ }^{66}$, W. Tkaczyk ${ }^{73}$, C.J. Todero Peixoto ${ }^{18,24}$, B. Tomé ${ }^{74}$, A. Tonachini ${ }^{53}$, P. Travnicek $^{29}$, D.B. Tridapalli ${ }^{19}$,

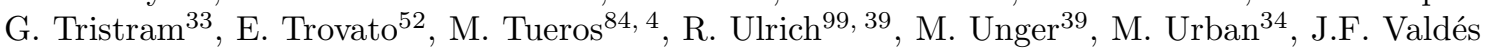
Galicia $^{67}$, I. Valiño ${ }^{84,39}$, L. Valore ${ }^{50}$, A.M. van den Berg $^{69}$, E. Varela ${ }^{63}$, B. Vargas Cárdenas ${ }^{67}$, J.R. Vázquez ${ }^{81}$, R.A. Vázquez ${ }^{84}$, D. Veberičc ${ }^{79}{ }^{78}$, V. Verzi ${ }^{51}$, J. Vicha ${ }^{29}$, M. Videla ${ }^{10}$, L. Villaseñor $^{66}$, H. Wahlberg ${ }^{7}$, P. Wahrlich ${ }^{13}$, O. Wainberg ${ }^{2}$, D. Warner ${ }^{91}$, A.A. Watson ${ }^{87}$, M. Weber ${ }^{40}$, K. Weidenhaupt ${ }^{43}$, A. Weindl ${ }^{39}$, S. Westerhoff ${ }^{108}$, B.J. Whelan ${ }^{13}$, G. Wieczorek ${ }^{73}$, L. Wiencke ${ }^{90}$, B. Wilczyńska ${ }^{72}$, H. Wilczyński ${ }^{72}$, M. Will ${ }^{39}$, C. Williams ${ }^{101}$, T. Winchen ${ }^{43}$, L. Winders ${ }^{109}$, M.G. Winnick ${ }^{13}$, M. Wommer ${ }^{39}$, B. Wundheiler ${ }^{2}$, T. Yamamoto ${ }^{101}$ a, T. Yapici ${ }^{95}$, P. Younk ${ }^{45}$, G. Yuan ${ }^{94}$, A. Yushkov ${ }^{84,50}$, B. Zamorano ${ }^{83}$, E. Zas ${ }^{84}$, D. Zavrtanik ${ }^{79,78}$, M. Zavrtanik ${ }^{78,79}$, I. Zaw ${ }^{96}$, A. Zepeda ${ }^{64}$, M. Ziolkowski ${ }^{45}$

${ }^{1}$ Centro Atómico Bariloche and Instituto Balseiro (CNEA- UNCuyo-CONICET), San Carlos de Bariloche, Argentina

${ }^{2}$ Centro Atómico Constituyentes (Comisión Nacional de Energía Atómica/CONICET/UTN-FRBA), Buenos Aires, Argentina

${ }^{3}$ Centro de Investigaciones en Láseres y Aplicaciones, CITEFA and CONICET, Argentina

${ }^{4}$ Departamento de Física, FCEyN, Universidad de Buenos Aires y CONICET, Argentina

${ }^{7}$ IFLP, Universidad Nacional de La Plata and CONICET, La Plata, Argentina

${ }^{8}$ Instituto de Astronomía y Física del Espacio (CONICET- UBA), Buenos Aires, Argentina 
${ }^{9}$ Instituto de Física de Rosario (IFIR) - CONICET/U.N.R. and Facultad de Ciencias Bioquímicas y Farmacéuticas U.N.R., Rosario, Argentina

${ }^{10}$ National Technological University, Faculty Mendoza (CONICET/CNEA), Mendoza, Argentina

${ }^{11}$ Pierre Auger Southern Observatory, Malargüe, Argentina

${ }^{12}$ Pierre Auger Southern Observatory and Comisión Nacional de Energía Atómica, Malargüe, Argentina

${ }^{13}$ University of Adelaide, Adelaide, S.A., Australia

${ }^{16}$ Centro Brasileiro de Pesquisas Fisicas, Rio de Janeiro, RJ, Brazil

${ }_{17}$ Pontifícia Universidade Católica, Rio de Janeiro, RJ, Brazil

18 Universidade de São Paulo, Instituto de Física, São Carlos, SP, Brazil

19 Universidade de São Paulo, Instituto de Física, São Paulo, SP, Brazil

${ }^{20}$ Universidade Estadual de Campinas, IFGW, Campinas, SP, Brazil

${ }^{21}$ Universidade Estadual de Feira de Santana, Brazil

${ }^{22}$ Universidade Estadual do Sudoeste da Bahia, Vitoria da Conquista, BA, Brazil

${ }^{23}$ Universidade Federal da Bahia, Salvador, BA, Brazil

${ }^{24}$ Universidade Federal do ABC, Santo André, SP, Brazil

${ }^{25}$ Universidade Federal do Rio de Janeiro, Instituto de Física, Rio de Janeiro, RJ, Brazil

${ }^{26}$ Universidade Federal Fluminense, EEIMVR, Volta Redonda, RJ, Brazil

27 Rudjer Bošković Institute, 10000 Zagreb, Croatia

28 Charles University, Faculty of Mathematics and Physics, Institute of Particle and Nuclear Physics, Prague, Czech Republic

${ }^{29}$ Institute of Physics of the Academy of Sciences of the Czech Republic, Prague, Czech Republic

${ }^{30}$ Palacky University, RCATM, Olomouc, Czech Republic

32 Institut de Physique Nucléaire d'Orsay (IPNO), Université Paris 11, CNRS-IN2P3, Orsay, France

33 Laboratoire AstroParticule et Cosmologie (APC), Université Paris 7, CNRS-IN2P3, Paris, France

${ }^{34}$ Laboratoire de l'Accélérateur Linéaire (LAL), Université Paris 11, CNRS-IN2P3, Orsay, France

${ }^{35}$ Laboratoire de Physique Nucléaire et de Hautes Energies (LPNHE), Universités Paris 6 et Paris 7, CNRS-IN2P3, Paris, France

${ }^{36}$ Laboratoire de Physique Subatomique et de Cosmologie (LPSC), Université Joseph Fourier, INPG, CNRS-IN2P3, Grenoble, France

37 SUBATECH, CNRS-IN2P3, Nantes, France

38 Bergische Universität Wuppertal, Wuppertal, Germany

${ }^{39}$ Karlsruhe Institute of Technology - Campus North - Institut für Kernphysik, Karlsruhe, Germany

${ }^{40}$ Karlsruhe Institute of Technology - Campus North - Institut für Prozessdatenverarbeitung und Elektronik, Karlsruhe, Germany

${ }^{41}$ Karlsruhe Institute of Technology - Campus South - Institut für Experimentelle Kernphysik (IEKP), Karlsruhe, Germany

${ }^{42}$ Max-Planck-Institut für Radioastronomie, Bonn, Germany

${ }^{43}$ RWTH Aachen University, III. Physikalisches Institut A, Aachen, Germany

${ }^{44}$ Universität Hamburg, Hamburg, Germany

${ }^{45}$ Universität Siegen, Siegen, Germany

${ }^{46}$ Dipartimento di Fisica dell'Università and INFN, Genova, Italy

${ }^{47}$ Università dell'Aquila and INFN, L'Aquila, Italy

48 Università di Milano and Sezione INFN, Milan, Italy

${ }^{49}$ Dipartimento di Fisica dell'Università del Salento and Sezione INFN, Lecce, Italy

${ }^{50}$ Università di Napoli "Federico II" and Sezione INFN, Napoli, Italy 
${ }^{51}$ Università di Roma II "Tor Vergata" and Sezione INFN, Roma, Italy

${ }^{52}$ Università di Catania and Sezione INFN, Catania, Italy

${ }^{53}$ Università di Torino and Sezione INFN, Torino, Italy

${ }^{54}$ Dipartimento di Ingegneria dell'Innovazione dell'Università del Salento and Sezione INFN, Lecce, Italy

${ }^{56}$ Istituto di Astrofisica Spaziale e Fisica Cosmica di Palermo (INAF), Palermo, Italy

${ }^{57}$ Istituto di Fisica dello Spazio Interplanetario (INAF), Università di Torino and Sezione INFN, Torino, Italy

${ }^{58}$ INFN, Laboratori Nazionali del Gran Sasso, Assergi (L'Aquila), Italy

${ }^{61}$ Università di Palermo and Sezione INFN, Catania, Italy

${ }^{63}$ Benemérita Universidad Autónoma de Puebla, Puebla, Mexico

${ }^{64}$ Centro de Investigación y de Estudios Avanzados del IPN (CINVESTAV), México, D.F., Mexico

${ }^{66}$ Universidad Michoacana de San Nicolas de Hidalgo, Morelia, Michoacan, Mexico

${ }^{67}$ Universidad Nacional Autonoma de Mexico, Mexico, D.F., Mexico

${ }^{68}$ IMAPP, Radboud University, Nijmegen, Netherlands

${ }^{69}$ Kernfysisch Versneller Instituut, University of Groningen, Groningen, Netherlands

70 NIKHEF, Amsterdam, Netherlands

71 ASTRON, Dwingeloo, Netherlands

72 Institute of Nuclear Physics PAN, Krakow, Poland

${ }^{73}$ University of Łódź, Łódź, Poland

${ }^{74}$ LIP and Instituto Superior Técnico, Lisboa, Portugal

78 J. Stefan Institute, Ljubljana, Slovenia

${ }^{79}$ Laboratory for Astroparticle Physics, University of Nova Gorica, Slovenia

${ }^{80}$ Instituto de Física Corpuscular, CSIC-Universitat de València, Valencia, Spain

${ }^{81}$ Universidad Complutense de Madrid, Madrid, Spain

${ }^{82}$ Universidad de Alcalá, Alcalá de Henares (Madrid), Spain

${ }^{83}$ Universidad de Granada \& C.A.F.P.E., Granada, Spain

${ }^{84}$ Universidad de Santiago de Compostela, Spain

${ }^{85}$ Rudolf Peierls Centre for Theoretical Physics, University of Oxford, Oxford, United Kingdom

87 School of Physics and Astronomy, University of Leeds, United Kingdom

${ }_{88}$ Argonne National Laboratory, Argonne, IL, USA

${ }^{89}$ Case Western Reserve University, Cleveland, OH, USA

${ }^{90}$ Colorado School of Mines, Golden, CO, USA

${ }^{91}$ Colorado State University, Fort Collins, CO, USA

${ }^{92}$ Colorado State University, Pueblo, CO, USA

${ }^{93}$ Fermilab, Batavia, IL, USA

${ }^{94}$ Louisiana State University, Baton Rouge, LA, USA

${ }^{95}$ Michigan Technological University, Houghton, MI, USA

${ }^{96}$ New York University, New York, NY, USA

${ }^{97}$ Northeastern University, Boston, MA, USA

${ }^{98}$ Ohio State University, Columbus, OH, USA

${ }^{99}$ Pennsylvania State University, University Park, PA, USA

100 Southern University, Baton Rouge, LA, USA

${ }^{101}$ University of Chicago, Enrico Fermi Institute, Chicago, IL, USA

105 University of Nebraska, Lincoln, NE, USA

106 University of New Mexico, Albuquerque, NM, USA

108 University of Wisconsin, Madison, WI, USA

109 University of Wisconsin, Milwaukee, WI, USA

${ }^{110}$ Institute for Nuclear Science and Technology (INST), Hanoi, Vietnam 
$(\dagger)$ Deceased

(a) at Konan University, Kobe, Japan

\begin{abstract}
The Pierre Auger Collaboration has reported evidence for anisotropy in the distribution of arrival directions of the cosmic rays with energies $E>E_{t h}=5.5 \times 10^{19} \mathrm{eV}$. These show a correlation with the distribution of nearby extragalactic objects, including an apparent excess around the direction of Centaurus A. If the particles responsible for these excesses at $E>E_{t h}$ are heavy nuclei with charge $Z$, the proton component of the sources should lead to excesses in the same regions at energies $E / Z$. We here report the lack of anisotropies in these directions at energies above $E_{t h} / Z$ (for illustrative values of $Z=6,13,26$ ). If the anisotropies above $E_{t h}$ are due to nuclei with charge $Z$, and under reasonable assumptions about the acceleration process, these observations imply stringent constraints on the allowed proton fraction at the lower energies.
\end{abstract}




\section{Introduction}

Anisotropy and composition, together with the study of the features in the energy spectrum, are the fundamental tools available to decipher the origin and nature of the ultra-high energy cosmic rays (UHECRs). The suppression of the flux observed above $40 \mathrm{EeV}$ 1, 2] suggests that the energy of the UHECRs is attenuated by interactions with the cosmic microwave background and infrared photons on their journey from their extragalactic sources, either by photopion interactions in the case of protons or by photodisintegration in the case of nuclei 3, 4. This would imply that at the highest energies cosmic rays can only arrive from nearby sources, within the so-called GZK horizon (which is e.g. $\sim 200 \mathrm{Mpc}$ for protons above $60 \mathrm{EeV}$ [5, 6]). This is supported by the correlation reported by the Pierre Auger Collaboration [7, 8, 9] between the arrival directions of cosmic rays with energies above $55 \mathrm{EeV}$ and the distribution of nearby extragalactic objects. The correlation with nearby active galactic nuclei (AGN) in the Véron-Cetty and Véron (VCV) catalog [10] was originally found with data collected up to May 2006, and was most significant for the AGN within $75 \mathrm{Mpc}$ and for angular separations between the AGN and cosmic ray arrival directions smaller than $3.1^{\circ}$. A test with subsequent data rejected the null hypothesis of isotropy with $99 \%$ confidence 7,8 . A more recent analysis 9 , has found that the fraction of events above $55 \mathrm{EeV}$ correlating with these AGN is $\left(38_{-6}^{+7}\right) \%$, smaller than obtained initially but still well above the isotropic expectation of $21 \%$. Note that these AGN may well be acting just as tracers of the actual UHECR sources, and indeed it is interesting that alternative studies with other populations (X-ray AGN from the SWIFT catalog or galaxies from the 2MASS catalog) also indicate some degree of correlation within a few degrees with those objects [9] (see also [11, 12, 13, 14, 15, 16]). The final identification of the UHECR sources will require much additional data.

Another interesting cosmic ray excess was found in the direction towards Cen A, at equatorial coordinates $(\alpha, \delta)=\left(201.4^{\circ},-43.0^{\circ}\right)$. Already in [7, 8] it was pointed out that two out of the 27 highest energy events observed before August 2007 by the Pierre Auger Observatory arrived within less than $3^{\circ}$ of Cen A, with several more events lying in the vicinity of its radio lobes. More recently, with data up to the end of 2009 [9] and considering the events above $55 \mathrm{EeV}$, the most significant excess around Cen A was identified for an $18^{\circ}$ window, in which 13 events were observed while only 3.2 were expected. Whether this excess, if confirmed with further data, is due to Cen A, which is one of the nearest AGN (being at less than $4 \mathrm{Mpc}$ distance), or due to one or several sources farther away, e.g. in the Centaurus cluster lying in a similar direction but at $\sim 45 \mathrm{Mpc}$, is something that remains to be determined. It should be mentioned that the HiRes air shower experiment has not found indications of an excess correlation with nearby AGN [17, although the associated statistics are smaller and there are systematic differences in the energy calibrations between the two experiments. Also, contrary to Auger, the HiRes experiment looks to the northern hemisphere, and in particular this makes it blind to the Cen A region of the sky.

The Pierre Auger Observatory has recently measured the average depth of the maximum of shower development $X_{\max }$ and its fluctuations [18. The logarithmic slope of the average shower maximum vs. energy becomes smaller above $\sim 2 \mathrm{EeV}$, indicating a change in the shower properties. Also the fluctuations in $X_{\max }$ become suppressed above this energy. An inference of the chemical composition of the primary cosmic rays can be done via comparison with Monte Carlo simulations of air showers. If these models are taken at face value, they indicate a gradual increase in the average mass as a function of energy 1 . Alternatively, this behavior could be ascribed to changes in the hadronic interactions (cross sections, inelasticities or multiplicities) not considered in the available models. We note that the models make extrapolations to energies well beyond those tested at accelerators. One should also keep in mind that, due to the limited statistics of the

\footnotetext{
${ }^{1}$ We note that the HiRes experiment measures a depth of shower maximum consistent with proton-only Monte Carlo air shower simulations all the way from $1 \mathrm{EeV}$ up to $\sim 40 \mathrm{EeV}$ [19].
} 
events observed with fluorescence telescopes, there is no measurement of the mean $X_{\max }$ and the corresponding fluctuations available for $E>55 \mathrm{EeV}$. It is clear that performing alternative studies to try to improve our understanding of the UHECR composition is important.

In this work we perform searches for anisotropies in the same directions where excesses were observed above $E_{t h}=55 \mathrm{EeV}$, but using lower energy thresholds (we consider the illustrative values for the threshold $E_{t h} / Z$, with $\left.Z=6,13,26\right)$.

We first focus on the analysis of the region around Cen A, for which the most significant excess was found above $55 \mathrm{EeV}$ for an angular window of $18^{\circ}$ radius. We note that the location of the excess, the size of the angular window and the selected energy threshold are a posteriori, therefore new independent data would be required to assess the significance of the excess at high energies. However, we are already able to report the results of our search for anisotropies in the same region for the lower energy thresholds considered.

We also perform a similar search but looking for possible excesses in windows of $3.1^{\circ}$ around the VCV AGN within $75 \mathrm{Mpc}$, considering only the data after May 2006 so as to exclude those used to fix these parameters. We note that even above $55 \mathrm{EeV}$ the cosmic ray deflections in the galactic magnetic field are likely larger than a few degrees (especially if cosmic rays happen to be heavy nuclei). However, the VCV correlation does not imply that the objects in this catalog are the sources, nor that the typical deflections are smaller than the optimal correlation angle. Active galaxies in the VCV catalog trace the nearby large scale matter distribution, and that includes all types of candidate astrophysical sources, not only AGN and their subclasses. Deflections of cosmic ray trajectories could be larger, and still manifest an anisotropy through a correlation of a fraction of them within a few degrees of the VCV objects.

We then explore the possibility that the anisotropies at the highest energies might be due to heavy nuclei. Using our observations and following an idea proposed by Lemoine and Waxman [20], which exploits the fact that a high energy anisotropy due to nuclei of charge $Z$ should lead to an anisotropy in the same region of the sky at energies $Z$ times smaller due to the protons from the same sources, we are then able to constrain the allowed proton fraction at the source under different assumptions on the value of the nuclear charges responsible for the high energy excess.

\section{The Observatory and the dataset:}

The Pierre Auger Observatory is located near the town of Malargüe, Argentina, at a latitude of $35.25^{\circ} \mathrm{S}$. It is a hybrid detector, consisting of 24 fluorescence telescopes and a surface array of 1600 water Cherenkov detectors covering $\sim 3000 \mathrm{~km}^{2}$ (see [21, 22, for further details).

The data considered in the present work consists of the cosmic ray events with zenith angles $\theta<60^{\circ}$ detected by the surface array (which has an almost $100 \%$ duty cycle and hence collected the largest data set) since 1 January 2004 up to 31 December 2009. The array has been growing in size until the completion of the baseline design in mid 2008. In order to have an accurate estimate of the exposure and hence of the expected background in the different regions of the sky we have removed periods in which the data acquisition was unstable (the resulting livetime being $87 \%$ [21]) and applied a quality cut that requires that for any event the six detectors surrounding the detector having the largest signal be active at the time the event is recorded. Keeping track of the number of active detector configurations able to trigger such events at any time allows us to take into account the detector growth and dead times in the evaluation of the exposure. The isotropic expectation in an angular window $\Delta \Omega$ can be obtained as $N_{\text {iso }}=x N_{\text {tot }}$, where $x$ is the fraction of the exposure within the solid angle $\Delta \Omega$ and $N_{\text {tot }}$ is the total number of events.

The trigger efficency is $100 \%$ for $E>3 \mathrm{EeV}$, but at lower energies (we consider here events down to $E=55 \mathrm{EeV} / 26 \simeq 2.1 \mathrm{EeV}$ ) the trigger efficiency becomes smaller than unity and is zenith angle dependent. Hence, to obtain the isotropic expectations for the lower energy threshold 
considered we use a fit to the zenith angle distribution of the events, rather than the ideal exposure expectation $\mathrm{d} N \propto \sin \theta \cos \theta \mathrm{d} \theta$. We note that the detection efficiency below $3 \mathrm{EeV}$ may also depend on the composition of the cosmic rays, being actually smaller for lighter nuclei. This could slightly affect the predictions for the expected localized proton excesses for the lowest energy threshold considered, $E>2.1 \mathrm{EeV}$. We estimate that in this case the predictions are affected by no more than $2 \%$ by the possible differences in exposure (using the values in ref. [21]), and hence these effects can be safely neglected.

\section{Results}

\subsection{The Centaurus A excess}

We first consider the excess observed in the Cen A region for energies above the threshold $E_{t h}=$ $55 \mathrm{EeV}$. The cumulative number of events as a function of the angular distance from the direction of Cen A is plotted in Fig. 1 (to make the plot more readable we display the difference with respect to the average isotropic expectations).

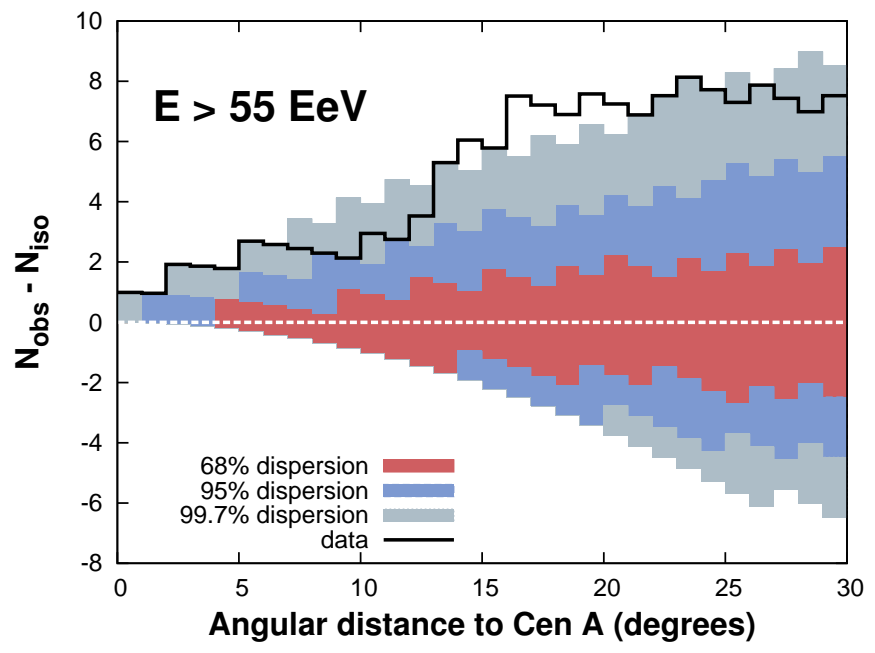

Figure 1: Cumulative number of events with $E \geq 55 \mathrm{EeV}$ (subtracting the average isotropic expectations) as a function of angular distance from the direction of Cen A. The bands correspond to the $68 \%, 95 \%$ and $99.7 \%$ dispersion expected for an isotropic flux.

In Fig. 2 we plot the cumulative number of events, subtracting the isotropic expectations, as a function of the angular distance from the direction of Cen A for lower energy thresholds, considering energies above $E_{t h} / Z$ in the cases $Z=6,13$ and 26. The observed distributions are consistent with the isotropic expectations (shaded regions), showing no significant excesses in any of the angular windows considered.

As reported in [9], the most significant excess for a top-hat window around Cen A was obtained for a radius $\gamma=18^{\circ}$ and we will hence focus on this region. For this energy range, the total number of event: 2 is $N_{\text {tot }}=60$, with $N_{\text {obs }}=10$ of these being in an $18^{\circ}$ angular window around Cen A. If we adopt the expression for the ideal exposure of the detector, the fraction of isotropic sky in

\footnotetext{
${ }^{2}$ Different from ref. [9, where 13 out of 69 events were reported to correlate within $18^{\circ}$ of Cen A, the stricter event selection applied in this work in order to get an accurate estimate of the exposure at low energies yields 10 correlations out of 60 events, well within the statistical uncertainties of the previous result.
} 
this $18^{\circ}$ region is $x \simeq 0.0466$. Normalizing to the counts outside the source region, the expected background in this region is $N_{b k g}=\left(N_{t o t}-N_{o b s}\right) x /(1-x)=2.44$ counts.
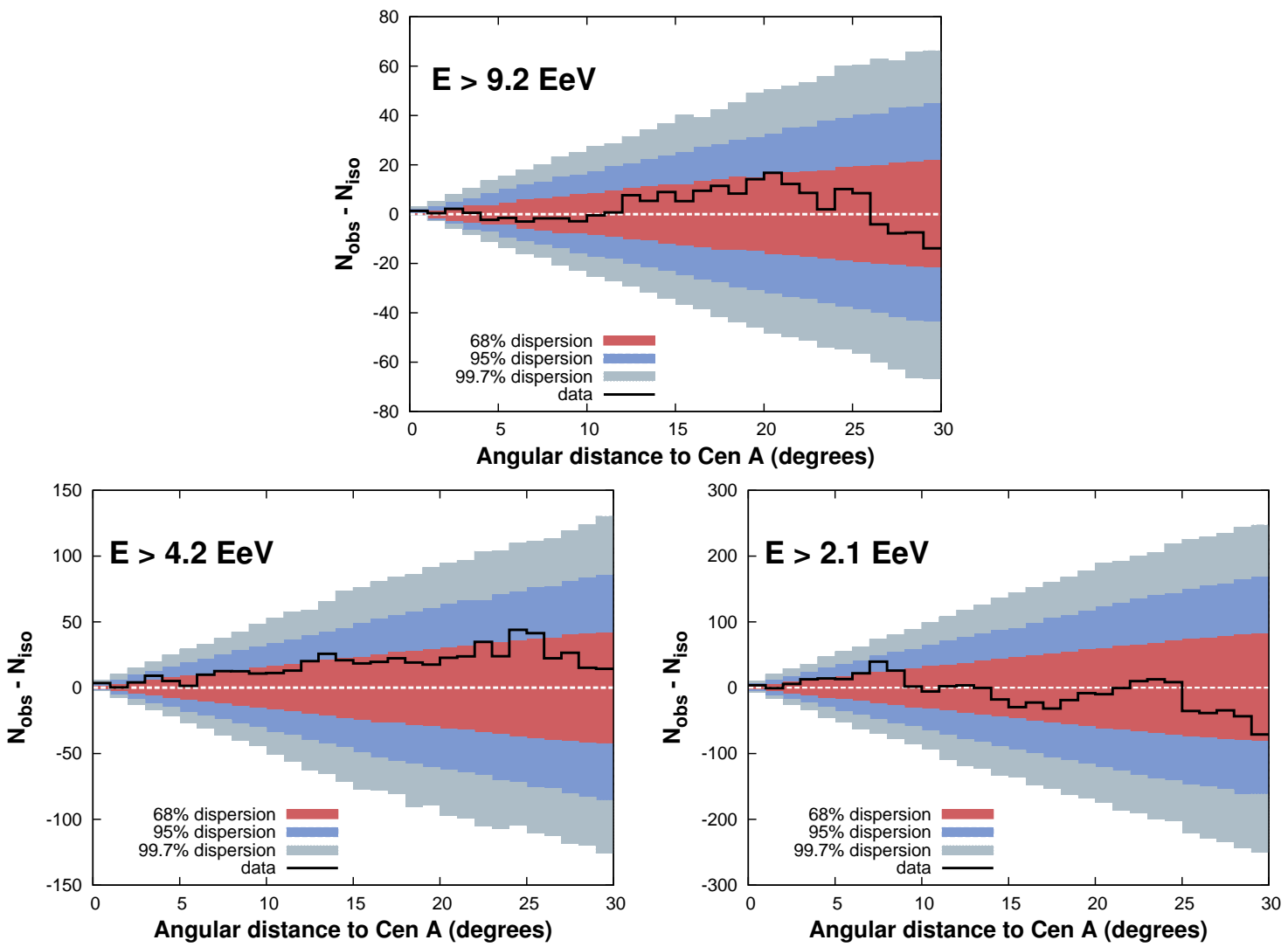

Figure 2: Similar to Fig. [1 events with $E \geq 55 \mathrm{EeV} / Z$ for $Z=6$ (top), 13 (bottom left) and 26 (bottom right).

In table 1 we report the observed number of events with $E>55 \mathrm{EeV} / \mathrm{Z}$ (total and in an angular window of $18^{\circ}$ around Cen A), as well as the expected isotropic background. No significant excess is found for any of the lower energy thresholds considered.

\begin{tabular}{|c|c||c|c|c|}
\hline$Z$ & $E_{\min }[\mathrm{EeV}]$ & $N_{\text {tot }}$ & $N_{\text {obs }}$ & $N_{\text {bkg }}$ \\
\hline \hline 6 & 9.2 & 4455 & 219 & $207 \pm 14$ \\
\hline 13 & 4.2 & 16640 & 797 & $774 \pm 28$ \\
\hline 26 & 2.1 & 63600 & 2887 & $2920 \pm 54$ \\
\hline
\end{tabular}

Table 1: Total number of events, $N_{t o t}$, and those observed in an angular window of $18^{\circ}$ around Cen A, $N_{o b s}$, as well as the expected background $N_{b k g}$. Results are given for different energy thresholds, corresponding to $E_{\min }=E_{t h} / Z$ for the indicated values of $Z$ and $E_{t h}=55 \mathrm{EeV}$.

\subsection{The VCV AGN}

We now search for possible overdensities of cosmic rays with arrival directions within $3.1^{\circ}$ of objects with redshift $z \leq 0.018(\sim 75 \mathrm{Mpc})$ in the VCV catalog. We use for this study only data 
collected after May 2006, subsequent to data used to specify the parameters that optimized the VCV correlation in that period.

In this case, one has that for $E>E_{t h}=55 \mathrm{EeV}$ there are $N_{t o t}=49$ events, of which $N_{\text {obs }}=20$ are within $3.1^{\circ}$ of the nearby AGN. On the other hand, the probability that isotropic cosmic rays correlate by chance with those objects is $x \simeq 0.212$ and hence $N_{b k g}=\left(N_{t o t}-N_{o b s}\right) x /(1-x)=7.88$.

In table 2 we show the observed number of events with $E>55 \mathrm{EeV} / Z$ (total and those within $3.1^{\circ}$ of an object with $z \leq 0.018$ in the VCV catalog), as well as the expected background. It is apparent that no significant excess is found for any of the lower energy thresholds considered.

\begin{tabular}{|c|c||c|c|c|}
\hline$Z$ & $E_{\text {min }}[\mathrm{EeV}]$ & $N_{\text {tot }}$ & $N_{\text {obs }}$ & $N_{\text {bkg }}$ \\
\hline \hline 6 & 9.2 & 3626 & 763 & $770 \pm 28$ \\
\hline 13 & 4.2 & 13482 & 2852 & $2860 \pm 54$ \\
\hline 26 & 2.1 & 51641 & 10881 & $10966 \pm 105$ \\
\hline
\end{tabular}

Table 2: Total number of events, $N_{\text {tot }}$, and those observed within $3.1^{\circ}$ from objects with $z \leq 0.018$ in the VCV catalog, $N_{o b s}$, as well as the expected isotropic background $N_{b k g}$. Results are given for different energy thresholds, corresponding to $E_{\min }=E_{t h} / Z$ for the indicated values of $Z$ and $E_{t h}=55 \mathrm{EeV}$.

\section{Constraints on the source composition}

As a by-product of the observations described above, and under reasonable assumptions on the cosmic ray acceleration and propagation, it is possible to set some constraints on the composition of the cosmic rays responsible for localized overdensities observed above $E_{t h}$. In order to do this, we elaborate on an idea proposed by Lemoine and Waxman [20], who related the high energy excess, under the assumption that it is due to heavy nuclei of charge $Z$, with the expected excess at energies above $E_{t h} / Z$ due to the protons from the same sources. Note that, in the absence of energy losses and scattering effects, protons with energies $E / Z$ would follow the same trajectories as nuclei of charge $Z$ and energy $E$ coming from the same source, and hence they should arrive within the same angular windows. Moreover, even if at lower energies the isotropic background can be enhanced by the contribution from sources beyond the GZK horizon, the gain in statistics obtained can make the search sensitive to relatively small low energy anisotropies.

The main underlying hypothesis is that the cosmic ray acceleration depends just on the particle rigidities, i.e. on $E / Z$. It is therefore natural to assume that at the sources the spectra of the different charge components scale as

$$
\frac{\mathrm{d} n_{Z}}{\mathrm{~d} E}=k_{Z} \Phi(E / Z),
$$

with $k_{Z}$ being constant factors. The function $\Phi$ may display a high energy cutoff resulting from the maximum rigidities attainable by the acceleration process. If, in this scenario, the maximum proton energies were below $E_{t h}$, the higher energy cosmic rays from the source could be dominated by a heavy component.

If $N(>E)$ is the number of events with energies above the threshold $E$ which come within a certain solid angle around a source and if the acceleration process at the source depends only on rigidity, then the number of nuclei of charge $Z$ above $E_{t h}$ and those of protons above $E_{t h} / Z$ are related by

$$
N_{p}\left(>E_{t h} / Z\right)=\frac{k_{p}}{Z k_{Z}} N_{Z}\left(>E_{t h}\right) .
$$


This relation does not take energy losses into account (included as a parameter $\alpha$ in ref. [20]). Ignoring them leads to more conservative bounds on the ratio $k_{p} / k_{Z}$, because energy losses are larger for nuclei of charge $Z$ and energy $E$ than for protons of energy $E / Z$. Moreover, the nucleons emitted in the photodisintegration processes can also add to the expected proton anisotropies at low energies.

The number of events produced by the source(s) responsible for the localized excess observed can be estimated as $N=N_{o b s}-N_{b k g}$ in terms of the number of events observed in the window considered and the expected background, which are displayed in the tables. Taking into account the Poisson fluctuations in the low and high energy signals, as well as in the background estimates, we obtain $95 \% \mathrm{CL}$ upperbounds on the quantity $R_{Z} \equiv N\left(>E_{t h} / Z\right) / N\left(>E_{t h}\right)$ using the profile likelihood method (see e.g. [23]). In the case of Cen A these bounds are $R_{Z}<12.9,17.3$ and 9.1 for $Z=26,13$ and 6 respectively, while for the case of VCV the bounds are $R_{Z}<14.7,12.4$ and 6.0. We note that considering a $99 \%$ confidence level, the bounds become $R_{Z}<23.8,31.1$ and 16.3 for Cen A, and $R_{Z}<28.9,23.7$ and 11.4 for $\mathrm{VCV}$ (for $Z=26,13$ and 6 respectively), being then typically a factor of two weaker.

If the excess at high energies is indeed dominated by the heavier nuclear component of charge $Z$, i.e. $N\left(>E_{t h}\right) \simeq N_{Z}\left(>E_{t h}\right)$, we obtain that $R_{Z}>N_{p}\left(>E_{t h} / Z\right) / N_{Z}\left(>E_{t h}\right)+1=k_{p} /\left(Z k_{Z}\right)+1$ (where we used that $N_{Z}\left(>E_{t h} / Z\right) / N_{Z}\left(>E_{t h}\right)>1$ ). In this way, conservative bounds $k_{p} / k_{Z}<$ $Z\left(R_{Z}-1\right)$ can be obtained.

One may translate these limits on the relative spectrum normalizations into bounds on the actual low energy abundance ratios between the proton and heavy elements at the source. In particular, in the case that one assumes that below a certain rigidity the spectrum has a power law behavior, i.e. $\Phi \propto(E / Z)^{-s}$ for $E / Z<E_{1}$, as expected in scenarios of diffusive shock acceleration, at energies below $E_{1}$ all the relative abundances of the different elements present will be independent of the energy. In this case, one can relate the low energy relative fractions $f_{i}$ of the different elements at the source with the normalization factors $k_{i}$ in eq. (11). Comparing the differential spectra for protons and for the charge $Z$ at energies below $E_{1}$ one gets

$$
\frac{k_{p}}{k_{Z}}=\frac{f_{p}}{f_{Z}} Z^{s}
$$

Note that we are not making any assumption about the spectral shape above the threshold energies. Also, for energies above $E_{1}$ the values of $f_{p} / f_{Z}$ will depend on the spectral shape details.

The resulting bounds for the low energy relative abundances are displayed in Figs. 3 and 4 as a function of the low energy spectral index, for values $1.5<s<2.5$. The regions above the respective lines are excluded at $95 \%$ CL.

In the case in which energy losses can be neglected, such as if the source of the excess events is the nearby Cen A galaxy, it is appropriate to consider energy bins and relate, through an expression analogous to that in eq. (2), the events in the bin $\left[E_{t h}, 2 E_{t h}\right]$ to those at $Z$ times lower energies (where we adopted for definiteness a bin width corresponding to a factor two in energy) 3 . The ratio between the events observed and those expected for the $18^{\circ}$ window around Cen $\mathrm{A}$ are $N_{\text {obs }} / N_{b k g}=152 / 153.5,543 / 533.4$ and $2090 / 2147.9$ for $Z=6,13$ and 26 respectively. This leads to bounds on the proton fractions similar to those in Fig. 3 but about a factor two stronger.

An important point is that the statistical significance of the constraints in Fig. 3 is a posteriori, since the identification of the region around Cen A, its angular size and the energy threshold were tuned to maximize this excess. Therefore it would be necessary to look to this same region using the same energy threshold with an independent dataset of comparable size so as to obtain an

\footnotetext{
${ }^{3}$ If energy losses were relevant, the observed energies of the high energy events might correspond to a wider span of energies at the source, and the corresponding low energy protons may then span a range of energies wider than $\left[E_{t h} / Z, 2 E_{t h} / Z\right]$, making this analysis no longer valid, while that based on the integral energy bins above a threshold would still provide conservative bounds.
} 


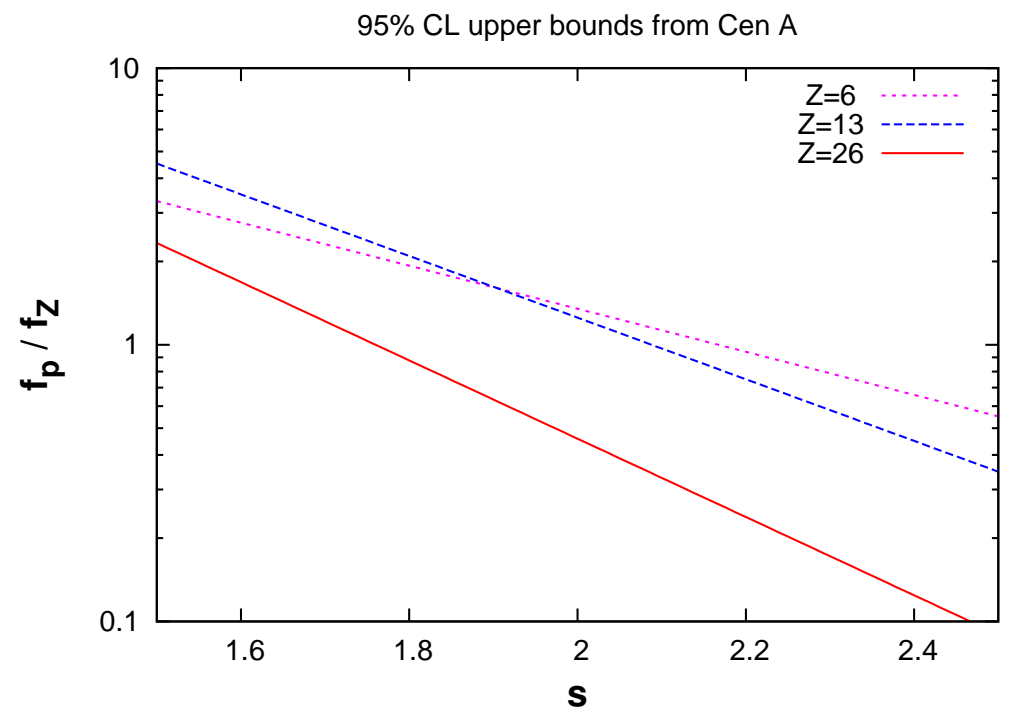

Figure 3: Upper bounds at 95\%CL on the allowed proton to heavy fractions in the source as a function of the assumed low energy spectral index $s$. The different lines are for charges $Z=6,13$ and 26 , as indicated.

unbiased estimate of the strength of the source (or sources) producing the excess. We note however that varying the energy threshold to 50 or $60 \mathrm{EeV}$ leads to qualitatively similar results. Also the angular size adopted for the window is not very crucial. For instance, if we consider a $10^{\circ}$ window instead of the $18^{\circ}$ one, the main effect on the bounds comes from the modification of the expected background in the low energy bin. This would relax the bounds on $f_{p} / f_{F e}$ by a factor of about two in this case. It is interesting to point out that the sensitivity of the a posteriori bounds from Cen A turns out to be comparable to that achieved with the analysis of the VCV correlations.

\section{Discussion and conclusions}

We have searched for overdensities at energies $E_{t h} / Z$ in the regions where anisotropies were reported previously above $E_{t h}=55 \mathrm{EeV}$, i.e. both in the direction towards Cen $\mathrm{A}$ and in $3.1^{\circ}$ windows around nearby AGN from the VCV catalog. Considering representative values of $Z=6$, 13 and 26 , where $Z$ is the assumed charge of the cosmic rays responsible for the high energy anisotropies, we have found no indications of overdensities in any of the lower energy bins. In scenarios where the acceleration process is only dependent on rigidity, the absence of significant anisotropies at energies $E>E_{t h} / Z$ implies that an upper bound can be set on the low-energy relative proton abundance at the sources. This bound is given by $f_{p} / f_{Z} \leq(0.5$ to 2$) Z^{2-s}$ at the 95\% CL level, depending on the adopted value of $Z$ (see lines in Figs. 3 and 4 ). Note that the constraints become weaker if the source spectrum is very hard $(s \simeq 1.5)$. Given the comparable bounds obtained for different values of $Z$, similar limits will result in the case in which the high energy anisotropy is dominated by nuclei belonging to a given mass group with similar values of $Z$.

On the other hand, estimates of the expected low energy relative abundances point towards values above these bounds. For instance, the ATIC-2 experiment [24] has measured that at $100 \mathrm{TeV}$ (the highest energies for which detailed composition measurements are available) one has $f_{p} \simeq$ 


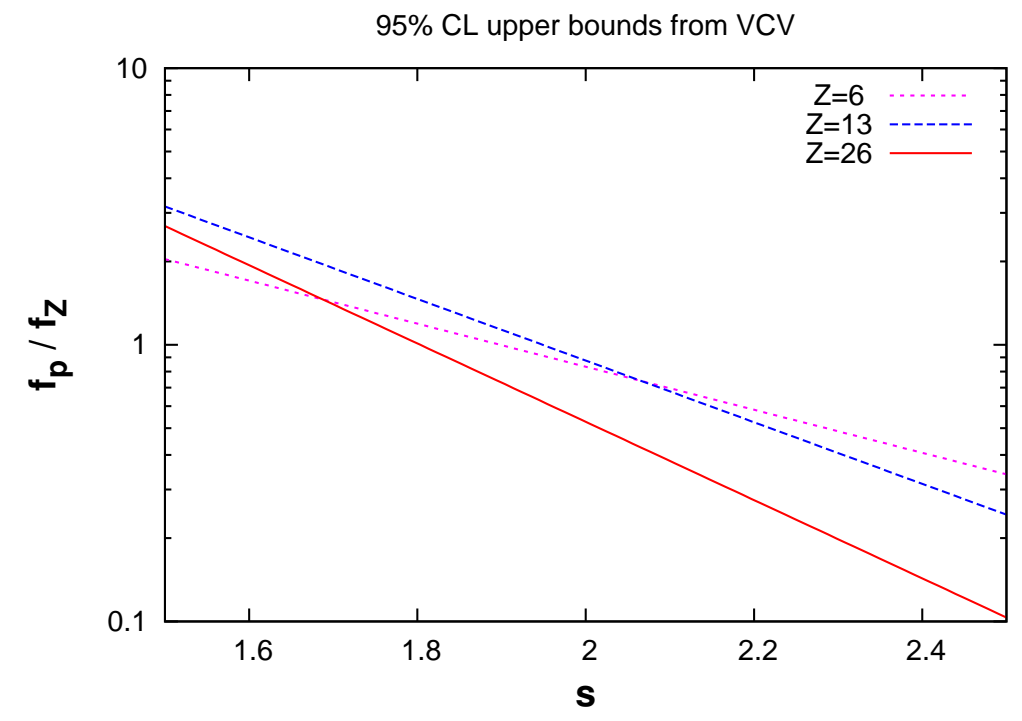

Figure 4: Upper bounds at 95\% CL on the allowed proton to heavy fractions in the source as a function of the assumed low energy spectral index $s$ implied by the absence of an excess of arrival directions of cosmic rays with energies above $55 \mathrm{EeV} / Z$ within $3.1^{\circ}$ of objects with $z \leq 0.018$ in the VCV catalog. The different lines are for charges $Z=6,13$ and 26, as indicated.

$f_{H e} \simeq 2 f_{C N O} \simeq 2 f_{N e-S i} \simeq 2 f_{Z>17} \simeq 4 f_{F e}$. Moreover, for these low energies (for which cosmic rays are believed to be of galactic origin) one would expect that the measured relative fraction of protons versus heavy nuclei for a given particle energy is actually smaller than the original fraction at the sources, due to the longer confinement time in the Galaxy of the heavier species. For instance, the measured $p$ to Fe fraction would be $26^{1 / 3} \simeq 3$ times smaller than the value at the source if the turbulent component of the galactic magnetic field has a Kolmogorov spectrum, so that the diffusion coefficient scales as $D \propto(E / Z)^{1 / 3}$. One has to keep in mind that these estimates based on lower energy galactic cosmic ray sources do not necessarily apply to the extragalactic sources which are most likely responsible for the highest energy events, but one may consider that they provide a useful indication of the plausible expected values.

Hence, we conclude that a heavy composition for the excesses observed at high energies appears to be in conflict with rigidity-dependent acceleration scenarios having at low energies a proton component more abundant than heavier species, as quantified in Fig. 4. How these conclusions are modified in the presence of strong structured magnetic fields and taking into account the relevant energy losses remains to be seen. We note that the present analysis based on the lack of anisotropies at lower energies provides information on the cosmic ray composition which is independent of $X_{\max }$ measurements, but depends instead on assumptions related to source properties.

\section{Acknowledgments}

The successful installation and commissioning of the Pierre Auger Observatory would not have been possible without the strong commitment and effort from the technical and administrative staff in Malargüe.

We are very grateful to the following agencies and organizations for financial support: Comisión Nacional de Energía Atómica, Fundación Antorchas, Gobierno De La Provincia de Mendoza, Mu- 
nicipalidad de Malargüe, NDM Holdings and Valle Las Leñas, in gratitude for their continuing cooperation over land access, Argentina; the Australian Research Council; Conselho Nacional de Desenvolvimento Científico e Tecnológico (CNPq), Financiadora de Estudos e Projetos (FINEP), Fundação de Amparo à Pesquisa do Estado de Rio de Janeiro (FAPERJ), Fundação de Amparo à Pesquisa do Estado de São Paulo (FAPESP), Ministério de Ciência e Tecnologia (MCT), Brazil; AVCR, AV0Z10100502 and AV0Z10100522, GAAV KJB300100801 and KJB100100904, MSMT-CR LA08016, LC527, 1M06002, and MSM0021620859, Czech Republic; Centre de Calcul IN2P3/CNRS, Centre National de la Recherche Scientifique (CNRS), Conseil Régional Ilede-France, Département Physique Nucléaire et Corpusculaire (PNC-IN2P3/CNRS), Département Sciences de l'Univers (SDU-INSU/CNRS), France; Bundesministerium für Bildung und Forschung (BMBF), Deutsche Forschungsgemeinschaft (DFG), Finanzministerium Baden-Württemberg, HelmholtzGemeinschaft Deutscher Forschungszentren (HGF), Ministerium für Wissenschaft und Forschung, Nordrhein-Westfalen, Ministerium für Wissenschaft, Forschung und Kunst, Baden-Württemberg, Germany; Istituto Nazionale di Fisica Nucleare (INFN), Istituto Nazionale di Astrofisica (INAF), Ministero dell'Istruzione, dell'Università e della Ricerca (MIUR), Gran Sasso Center for Astroparticle Physics (CFA), Italy; Consejo Nacional de Ciencia y Tecnología (CONACYT), Mexico; Ministerie van Onderwijs, Cultuur en Wetenschap, Nederlandse Organisatie voor Wetenschappelijk Onderzoek (NWO), Stichting voor Fundamenteel Onderzoek der Materie (FOM), Netherlands; Ministry of Science and Higher Education, Grant Nos. 1 P03 D 01430 and N N202 207238 , Poland; Fundação para a Ciência e a Tecnologia, Portugal; Ministry for Higher Education, Science, and Technology, Slovenian Research Agency, Slovenia; Comunidad de Madrid, Consejería de Educación de la Comunidad de Castilla La Mancha, FEDER funds, Ministerio de Ciencia e Innovación and Consolider-Ingenio 2010 (CPAN), Generalitat Valenciana, Junta de Andalucía, Xunta de Galicia, Spain; Science and Technology Facilities Council, United Kingdom; Department of Energy, Contract Nos. DE-AC02-07CH11359, DE-FR02-04ER41300, National Science Foundation, Grant No. 0969400, The Grainger Foundation USA; NAFOSTED, Vietnam; ALFA-EC / HELEN, European Union 6th Framework Program, Grant No. MEIF-CT-2005-025057, European Union 7th Framework Program, Grant No. PIEF-GA-2008-220240, and UNESCO.

\section{References}

[1] J. Abraham et al., The Pierre Auger Collaboration, Phys. Lett. B 685 (2010) 239.

[2] R. U. Abbasi et al., The HiRes Collaboration, Phys. Rev. Lett. 100 (2008) 101101.

[3] K. Greisen, Phys. Rev. Lett. 16 (1966) 748.

[4] G. T. Zatsepin, V. A. Kuz'min, Sov. Phys. JETP Lett. 4 (1966) 78.

[5] D. Harari, S. Mollerach, E. Roulet, JCAP 11 (2006) 012.

[6] A. V. Olinto, D. Allard, E. Armengaud, A. Kravtsov, Proc. 30 ${ }^{\text {th }}$ ICRC Vol. 4 (2008) 527.

[7] J. Abraham et al., The Pierre Auger Collaboration, Science 318 (2007) 938.

[8] J. Abraham et al., The Pierre Auger Collaboration, Astropart. Phys. 29 (2008) 188.

[9] P. Abreu et al., The Pierre Auger Collaboration, Astropart. Phys. 34 (2010) 314.

[10] M.-P. Véron-Cetty, P. Véron, Astron. \& Astrophys. 455 (2006) 773.

[11] T. Kashti, E. Waxman, JCAP 05 (2008) 006. 
[12] M. George, A. C. Fabian, W. H. Baumgartner, R. F. Mushotzky, J. Tueller, Month. Notices Royal Astron. Soc. 388 (2008) L59.

[13] G. Ghisellini, G. Ghirlanda, F. Tavecchio, F. Fraternali, G. Pareschi, Month. Notices Royal Astron. Soc. 390 (2008) L88.

[14] N. M. Nagar, J. Matulich, Astron. \& Astrophys. 488 (2008) 879.

[15] H. Takami, T. Nishimichi, K. Yahata, K. Sato, JCAP 06 (2009) 031.

[16] H. B. J. Koers, P. Tinyakov, JCAP 04 (2009) 003.

[17] R. U. Abbasi et al., The HiRes Collaboration, Astropart. Phys. 30 (2008) 175.

[18] J. Abraham et al., The Pierre Auger Collaboration, Phys. Rev. Lett. 104 (2010) 091101.

[19] R. U. Abbasi et al., The HiRes Collaboration, Phys. Rev. Lett. 104 (2010) 161101.

[20] M. Lemoine, E. Waxman, JCAP 11 (2009) 009.

[21] J. Abraham et al., The Pierre Auger Collaboration, Nucl. Instr. and Meth. in Physics Research A613 (2010) 29.

[22] J. Abraham et al., The Pierre Auger Collaboration, Nucl. Instr. and Meth. in Physics Research A620 (2010) 227.

[23] W. A. Rolke, A. M. López, J. Conrad, Nucl. Instrum. and Meth. in Physics Research A551 (2005) 493.

[24] A. Pavnov et al., (ATIC-2 Collaboration), Bull. Russ. Acad. Sc.: Physics 71 (2007) 494; ibidem Physics 73 (2009) 564. 